University of Nebraska - Lincoln

DigitalCommons@University of Nebraska - Lincoln

May 1974

\title{
Photoelectron Angular Distributions as a Probe of Anisotropic Electron-Ion Interactions
}

Dan Dill

Boston University, Boston, Massachusetts

Steven T. Manson

Georgia State University, Atlanta, Georgia

Anthony F. Starace

University of Nebraska-Lincoln, astarace1@unl.edu

Follow this and additional works at: https://digitalcommons.unl.edu/physicsstarace

Part of the Physics Commons

Dill, Dan ; Manson, Steven T.; and Starace, Anthony F., "Photoelectron Angular Distributions as a Probe of Anisotropic Electron-Ion Interactions" (1974). Anthony F. Starace Publications. 14.

https://digitalcommons.unl.edu/physicsstarace/14

This Article is brought to you for free and open access by the Research Papers in Physics and Astronomy at DigitalCommons@University of Nebraska - Lincoln. It has been accepted for inclusion in Anthony F. Starace Publications by an authorized administrator of DigitalCommons@University of Nebraska - Lincoln. 


\title{
PHYSICAL REVIEW LETTERS
}

\begin{tabular}{l}
\hline Volume 32 \\
\hline
\end{tabular}

\section{Photoelectron Angular Distributions as a Probe of Anisotropic Electron-Ion Interactions*}

\author{
Dan Dill \\ Department of Chemistry, Boston University, Boston, Massachusetts 02215 \\ and \\ Steven T. Manson \\ Department of Physics, Georgia State University, Atlanta, Georgia 30303 \\ and \\ Anthony F. Starace \\ Behlen Laboratory of Physics, The University of Nebraska, Lincoln, Nebraska 68508 \\ (Received 31 December 1973)

\begin{abstract}
Expressions are given for atomic photoelectron angular distributions in $L S$ coupling in which the role of anisotropic final state electron-ion interactions emerges explicitly. Calculations of photoelectron angular distributions for atomic sulfur are presented in which these anisotropic interactions produce clear deviations from the predictions of the Cooper-Zare model. Such effects are expected to be a general feature of photoelectron
\end{abstract} \\ angular distributions for most open-shell atoms.
}

We report here expressions for atomic photoelectron angular distributions in $L S$ coupling which exhibit clearly the influence of anisotropic electron-ion interactions. To illustrate these effects we have calculated the angular distributions of electrons photoionized from atomic sulfur, for which these anisotropic final-state interactions are large. These effects take the form of pronounced differences between the distributions of photoelectron groups corresponding to alternative $L S$-term levels of the residual ion. This result is to be contrasted with that of the Cooper-Zare (CZ) model, ${ }^{1}$ in which the role of final-state interactions is not considered: No dependence on the ionic term level is predicted. The past success of the $\mathrm{CZ}$ model in confirming measurements ${ }^{2,3}$ is due to the fortuitous circumstance that the measurements have dealt with closed-shell atoms, for which we show that angular momentum and parity conservation impose severe restrictions on the effects of any anisotropic interactions.

Our results are aimed on the one hand at theorists engaged in photoionization cross section calculations that include electron correlation. The criteria for assessing the importance of anisotropic interactions are given in terms of interaction parameters provided by such calculations. On the other hand, we wish to emphasize to experimentalists this new dynamical information on final-state interactions that can emerge through the study of open-shell atoms.

Our analysis is based on the resolution of the angular distribution into separate contributions characterized by the alternative values $j_{t}$ of the angular momentum transferred in the photoioni- 
zation process. ${ }^{4}$ Consider the following schematic photoionization process:

$$
A\left(J_{0} \pi_{0}\right)+\gamma\left(j_{\gamma}=1, \pi_{\gamma}=-1\right)-A^{+}\left(J_{c} \pi_{c}\right)+e^{-}\left(l s j, \pi_{e}=(-1)^{l}\right) .
$$

If no measurements are made of the orientation of the ion or of the polarization of the electron's spin, then the amplitudes for photoionization with alternative values of the angular momentum transfer $\overrightarrow{\mathrm{j}}_{t}=\overrightarrow{\mathrm{J}}_{c}+\overrightarrow{\mathrm{s}}-\overrightarrow{\mathrm{J}}_{0}=\overrightarrow{\mathrm{j}}_{\gamma}-\overrightarrow{\mathrm{I}}$ superpose incoherently in the differential cross section. ${ }^{5}$ The allowed values of $j_{t}$ are those consistent with the conservation of total angular momentum $\overrightarrow{\mathrm{J}}=\overrightarrow{\mathrm{J}}_{0}+\overrightarrow{\mathrm{j}}_{\gamma}=\overrightarrow{\mathrm{J}}_{c}$ $+\overrightarrow{\mathbf{S}}+\overrightarrow{\mathrm{I}}$ and parity $\pi=\pi_{0} \pi_{\gamma}=\pi_{c} \pi_{e}$. In the absence of all anisotropic interactions between the departing electron and the ion, however, $j_{t}$ is restricted to the single value $l_{0}$, the photoelectron's initial orbital momentum, and the resulting angular distribution is that given by the $\mathrm{CZ}$ model. ${ }^{4}$ Consequently, contributions to Reaction (1) by angular momentum transfers $j_{t} \neq l_{0}$ are a measure of both anisotropic interaction strength and the breakdown of the $\mathrm{CZ}$ model. This consideration motivated our analysis.

$$
A\left(\left(l_{0}^{n} L_{0} S_{0}\right) J_{0} \pi_{0}\right)+\gamma\left(j_{\gamma}=1, \pi_{\gamma}=-1\right) \rightarrow A^{+}\left(\left(l_{0}^{n-1} L_{c} S_{c}\right) J_{c} \pi_{c}\right)+e^{-}\left(l_{s j}, \pi_{e}=(-1)^{l}\right) .
$$

In particular, we are concerned with the dependence of the angular distribution on different ionic term levels, $L_{c} S_{c}$, for the usual circumstance where the separation between such levels is far greater than the separations between the fine-structure levels $J_{c}$ of a given term. For this situation the scattering amplitude for transfer of $j_{t}$ units of angular momentum is ${ }^{6}$

$$
\begin{aligned}
& S_{l}\left(j_{t}\right)=\frac{4 \pi}{\lambda}\left(\frac{\pi \alpha h \nu}{3}\right)^{1 / 2} i^{-l} \exp \left(i \sigma_{\epsilon l}\right) \hat{J}_{0} \hat{l} \hat{l}_{0}\left(\begin{array}{lll}
l & 1 & l_{0} \\
0 & 0 & 0
\end{array}\right)\left(l_{0}{ }^{n} L_{0} S_{0} \llbracket l_{0}{ }^{n-1} L_{c} S_{c}\right) \\
& \times \sum_{L} \exp \left(i \delta_{\epsilon l} L_{c} S{ }^{L}\right) R_{\epsilon l}{ }^{L}{ }^{c} s^{L}{ }^{L} \hat{L}^{2}\left\{\begin{array}{ccc}
L_{0} L_{c} & j_{t} \\
l & 1 & L
\end{array}\right\}\left\{\begin{array}{ccc}
L_{0} L_{c} & l_{0} \\
l & 1 & L
\end{array}\right\} .
\end{aligned}
$$

Here $\sigma_{\epsilon l}$ is the Coulomb phase shift, dependent on the photoelectron orbital momentum $l$ and kinetic energy $\epsilon, \hat{x} \equiv(2 x+1)^{1 / 2}, \nu \lambda=c, R_{\epsilon l}{ }^{L_{c}} S_{c} L$ is the radial dipole matrix element, and $\delta_{\epsilon l}{ }^{L_{c} S_{c} L}$ is the photoelectron phase shift relative to Coulomb waves.

The dependence of the phase shift $\delta_{\epsilon l}{ }_{c}{ }_{c} S_{c}{ }^{L}$ and dipole matrix element $R_{\epsilon l}{ }_{c} s_{c}{ }^{L}$ on the term level of the residual ion arises through the dynamical coupling of the orbital motion of the electron to the net orbital motion of the residual ion. ${ }^{6}$ This coupling determines dynamical weights with which transition amplitudes for alternative values of the total orbital momentum $\overrightarrow{\mathrm{L}}=\overrightarrow{\mathrm{L}}_{c}+\overrightarrow{\mathrm{I}}$ superpose in Eq. (5). When there is no dynamical coupling, the weights become independent of $L$, and hence of $L_{c}$ :

$$
\exp \left(i \delta_{\epsilon l} L_{c} S_{c} L\right) R_{\epsilon l}{ }^{L_{c}} S_{c} L \frac{}{\text { no inter action }} \exp \left(i \delta_{\epsilon l}\right) R_{\epsilon l}
$$

The remaining statistical weights can then be summed analytically:

$$
\sum_{L} \hat{L}^{2}\left\{\begin{array}{ccc}
L_{0} & L_{c} & j_{t} \\
l & 1 & L
\end{array}\right\}\left\{\begin{array}{ccc}
L_{0} & L_{c} & l_{0} \\
l & 1 & L
\end{array}\right\}=\hat{l}_{0}^{-2} \delta\left(j_{t}, l_{0}\right) .
$$

That is, only in the limit of vanishing anisotropic electron-ion interaction is the motion of the photoelectron independent of the term level of the ion; $j_{t}$ is restricted to the single value $j_{t}=l_{0}$, and, upon 
using Eqs. (5) - (7), the asymmetry parameter (3) reduces to the $\mathrm{CZ}$ formula. ${ }^{4}$

The scattering amplitudes enter into the expression for $\beta$ as $\left|S_{+}\left(j_{t}\right)\right|^{2},\left|S_{-}\left(j_{t}\right)\right|^{2}$, and $\left[S_{+}\left(j_{t}\right)\right.$ $\times S_{-}{ }^{\dagger}\left(j_{t}\right)+$ c.c.], where the subscripts plus and minus denote $l=j_{t} \pm 1$. While only the third of these terms will depend on the Coulomb phase shift difference $\sigma_{+}-\sigma_{-}$, all three terms depend on the interference between the different terms of (5), i.e., on the phase shift differences of alternative pairs of electron-ion $L S$-coupled channels $\left(L_{0} l\right) L$. In contrast, the CZ formula has only the single interference, in the third term, depending on the total phase shift difference $\left(\sigma_{+}\right.$ $\left.+\delta_{+}\right)-\left(\sigma_{-}+\delta_{-}\right)$between the two independent-particle-model channels $l=l_{0} \pm 1$. Thus, the differences between the phase shifts $\delta_{\epsilon l}{ }_{c}{ }_{c} S_{c}{ }^{L}$ for alternative channels $\left(L_{c} l\right) L$ measure the extent of anisotropic interactions and thus the validity of the CZ model.

The anisotropic electron-ion coupling thus results in an angular distribution which differs from the $\mathrm{CZ}$ result in two respects: (1) The asymmetry parameter depends on interference of ionization amplitudes characterized not only by alternative values of $\vec{i}$, but also by alternative values of the total orbital momentum $\overrightarrow{\mathrm{L}}$. (2) All allowed values of the angular momentum transfer can be expected to contribute to the ionization process.

However, both of these factors are inoperative in the special case of ionization from a closed shell-for then $L_{0}=0$ and the sum over $L$ in Eq. (5) collapses to the single term with $L=j_{\gamma}=1$, and $j_{t}$ is restricted to the single value $j_{t}=l_{0}$. Thus purely geometrical factors impose severe

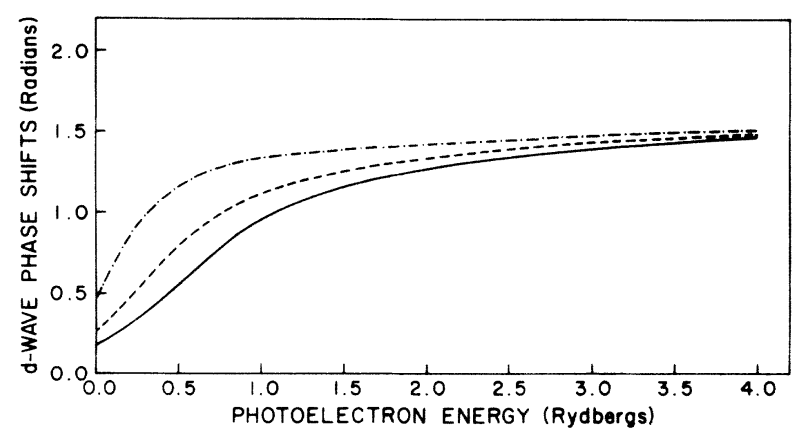

FIG。1. Hartree-Fock $d$-wave phase shifts $\delta_{\boldsymbol{\epsilon} d} L_{c} S_{c} L$ for the ${ }^{2} D^{0}$ ion of sulfur term versus photoelectron kinetic energy $\epsilon$ for alternative allowed values of $L$. Solid line, $L=0$ [i.e., the state $3 p^{3}\left({ }^{2} D\right) \epsilon d^{3} S$ ]; dashed line, $L$ $=1\left({ }^{3} P\right)$; dot-dashed line, $L=2\left({ }^{3} D\right)$. restrictions, consistent with the $\mathrm{CZ}$ model, on the angular distribution of photoelectrons ejected from closed shells, and it is for this reason that the $\mathrm{CZ}$ model has been generally successful when applied to such systems. ${ }^{2,3}$

To illustrate these ideas consider the photoionization of a typical open-shell atom such as sulfur:

$$
\mathrm{S}\left(3 p^{43} P\right)+\gamma \rightarrow \mathrm{S}^{+}\left(3 p^{3} L_{c} S_{c}\right)+e^{-}(l=0,2) .
$$

The allowed values of $L_{c} S_{c}$ are ${ }^{4} S^{0},{ }^{2} D^{0}$, and ${ }^{2} P^{0}$. Ionization to each of these terms can proceed with $j_{t}=l_{0}=1$, both for $l=0$ and for $l=2$. In addition, when $l=2$ the ${ }^{2} P^{0}$ term can also result from the transfer of $j_{t}=2$ units of angular momentum, and the ${ }^{2} D^{0}$ term has both $j_{t}=2$ and $j_{t}=3$ allowed. In Fig. 1 we plot the Hartree-Fock phase shifts $\delta_{\epsilon d} L_{c} S_{c}{ }^{L}$ as a function of photoelectron kinetic energy $\epsilon$ for the ${ }^{2} D^{0}$ ion term and for alternative allowed values of the total $L$. Because of the differences in these phase shifts we expect the predictions of the $\mathrm{CZ}$ model to be erroneous for sulfur.

Figure 2 shows our calculated asymmetry parameters for the three photoelectron groups belonging to the alternative ionic term levels as a function of $\epsilon$. The length formula for the dipole matrix elements has been used, since this is the correct one for Hartree-Fock calculations. ${ }^{7}$ As expected, contrary to the CZ model, these asymmetry parameters are found to be different from one another, particularly in the region of the Cooper minimum ${ }^{8}$ in the total cross section

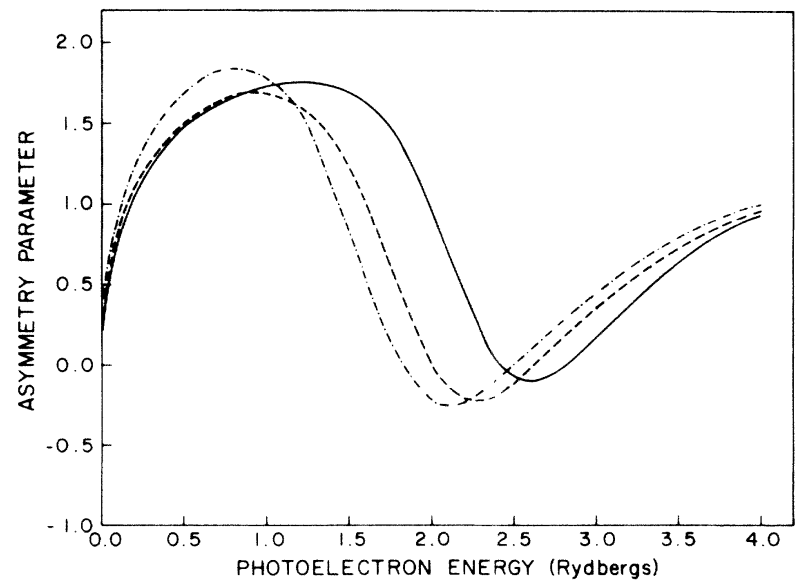

FIG。2. Asymmetry parameters $\beta\left(L_{c} s_{c}\right)$ for the photoionization transitions $3 p^{4}\left({ }^{3} P\right) \rightarrow 3 p^{3}\left(L_{c} S_{c}\right)+e^{-}$in sulfur versus photoelectron kinetic energy。 Solid line, ${ }^{4} S$ ionic term; dashed line, ${ }^{2} D$; dot-dashed line, ${ }^{2} P$. 


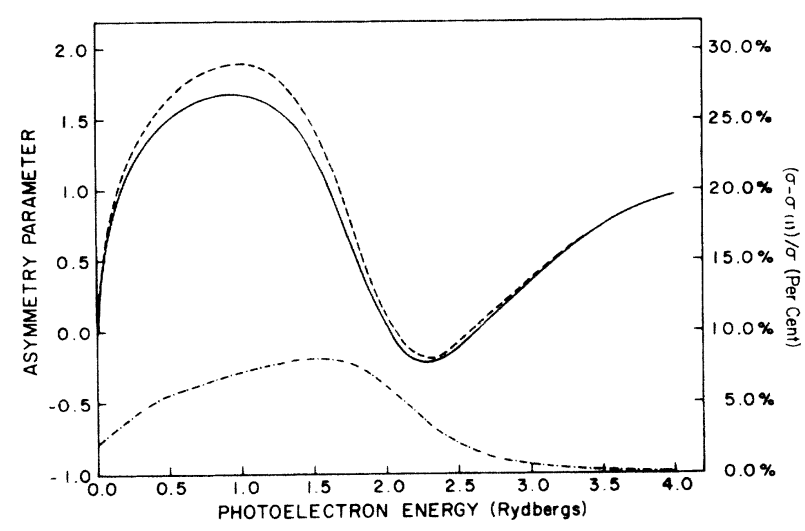

FIG. 3. Dependence of asymmetry parameter $\beta$ and cross section $\sigma$ for the ${ }^{2} D$ ion term of sulfur on angular momentum transfers $j_{t} \neq l_{0}$ as a function of photoelectron kinetic energy. Left-hand scale refers to (1) the solid line denoting $\beta$ and (2) the dashed line denoting $\beta\left(j_{t}=l_{0}=1\right)$. Right-hand scale refers to the dot-dashed line which denotes the ratio $\left[\sigma-\sigma\left(j_{t}=1\right)\right] / \sigma$.

(which is due to the sign change in the $3 p \rightarrow \epsilon d$ radial dipole matrix elements in the region $\epsilon \simeq 2$ Ry). A CZ model calculation, employing wave functions independent of $L S$ term structure, would produce a single asymmetry parameter (not shown in Fig. 2) for all three residual ion levels. Use of improved wave functions might shift the positions of the minima of our calculated $\beta$ parameters, but the differences between the three curves in Fig. 2 should remain.

Figure 3 shows the dependence of $\beta$ and of the total cross section $\sigma$ on angular momentum trans fers $j_{t} \neq l_{0}$, which do not occur in the CZ model. The solid line presents the same $\beta$ for the ${ }^{2} D^{0}$ ion level as in Fig. 2. The dashed line, however, is a plot of $\beta\left(j_{t}=1\right)$ for the ${ }^{2} D^{0}$ level, which would equal $\beta$ only if $\sigma\left(j_{t} \neq 1\right)=0$. We see that the difference $\beta-\beta\left(j_{t}=1\right)$ can be as large as 0.2 . The dot-dashed line in Fig. 3 is a plot of the percentage contribution of angular momentum transfers $j_{t} \neq 1$ to the total cross section. This percentage reaches a maximum of more than $8 \%$ for the ${ }^{2} D^{0}$ ion level.

In conclusion, we have presented criteria both for determining the importance of anisotropic electron-ion interactions and equivalently for establishing the validity of the $\mathrm{CZ}$ formula for the asymmetry parameter $\beta$. Namely, for most open-shell atoms we expect anisotropic interactions to exert significant effects, and therefore the $\mathrm{CZ}$ formula to give inaccurate predictions, whenever the phase shifts for different total angular momenta $L$ differ significantly from one another. Atomic sulfur has been presented as a typical example. Atomic oxygen and other first-row open-shell atoms are exceptions, having weak anisotropic interactions and therefore asymmetry parameters well predicted by the $\mathrm{CZ}$ formula. ${ }^{9}$ Detailed theoretical and numerical analyses of our results will be given elsewhere.$^{6.9}$

*Work supported in part by the U. S. Atomic Energy Commission under Contract No. COO-1674-88, by the National Science Foundation under Contract No. GP38905 , and by the National Aeronautics and Space Administration under Contract No. NGR 28-004-021.

${ }^{1} \mathrm{~J}$. Cooper and R. N. Zare, in Lectures in Theoretical Physics, edited by S. Geltman, K. T. Mahanthappa, and W. E. Brittin (Gordon and Breach, New York, 1969), Vol. XI-C, pp. 317-337.

${ }^{2}$ For representative measurements see, e.g., T. A. Carlson, G. E. McGuire, A. E. Jonas, K. L. Cheng, C. P. Anderson, C. C. Lu, and B. P. Pullen, in Proceedings of an International Conference on Electron Spectroscopy, Asilomar, Califormia, September 1971, edited by D. A. Shirley (North-Holland, Amsterdam, 1972), pp. 207-231; P. Mitchell and K. Codling, Phys. Lett. 38A, 31 (1972); M. J. Lynch, A. B. Gardner, and K. Codling, Phys. Lett. $\underline{40 \mathrm{~A}}, 349$ (1972); A. Niehaus and M. W. Ruf, Z. Phys. 252, 84 (1972); M. J. Lynch, K. Codling, and A. B. Gardner, Phys. Lett. 43A, 213 (1973); M. J. Van der Wiel and C. E. Brion, J. Electron Spectrosc. Relat. Phenomena 1, 739 (1973); G. R. Branton and C. E. Brion, to be published.

${ }^{3}$ For representative calculations see, e.g., J. W. Cooper and S. T. Manson, Phys. Rev. 177, 157 (1969); D. J. Kennedy and S. T. Manson, Phys. Rev. A $\underline{5}, 227$ (1972); S. T. Manson, J. Electron Spectrosc. Relat. Phenomena 1 , 413 (1973); S. T. Manson, Chem. Phys。 Lett. 19, 76 (1973).

${ }^{4}$ D. Dill, Phys. Rev. A $\underline{7}, 1976$ (1973), Sect. II.

${ }^{5}$ U. Fano and D. Dill, Phys. Rev. A 6, 185 (1972).

${ }^{6} \mathrm{D}$. Dill, S. T. Manson, and A. F. Starace, to be published.

${ }^{7}$ A. F. Starace, Phys. Rev。A $\underline{3}, 1242$ (1971), and $\underline{8}$, 1141 (1973).

${ }^{8}$ J. W. Cooper, Phys. Rev. 128, 681 (1962)。

${ }^{9}$ A. F. Starace, S. T. Manson, and D. J. Kennedy, Phys. Rev. A (to be published). 